\title{
ANÁLISIS EXPERIMENTAL DE COBERTURA EN UNA RED MALLADA PREDECIBLE APLICANDO MODELOS DE REGRESIÓN NO LINEAL EN AMBIENTES INTERIORES
}

\section{EXPERIMENTAL ANALYSIS OF COVERAGE ON A PREDICTABLE MESH NETWORK USING MODELS NONLINEAR REGRESSION INTERIOR ENVIRONMENTS}

\author{
MSc. Jorge Enrique Herrera Rubio, Ing. Carlos A. Hernández Sanabria. \\ Universidad de Pamplona, Campus Villa Rosario. \\ Ciudad Universitaria de la Frontera, Villa Rosario, Norte de Santander, Colombia. \\ Tel: (+577) 5703837 - $(+577) 5703742$. \\ E-mail: jherrera@unipamplona.edu.co, carlos.hernandezsanabria90@gmail.com
}

\begin{abstract}
Resumen: La metodología utilizada consiste en seleccionar y confinar el espacio de trabajo, determinar los criterios técnicos, la reglamentación y la capacidad de los equipos, registrar de los datos, ejecutar el proceso analítico, analizar los resultados con estadística y comparar con los modelos de estimación en ambientes interiores. El resultado esperado es obtener los coeficientes del modelo matemático que se aproxime a los niveles de potencia medidos y calculados. Finalmente confrontar los datos obtenidos con modelos establecidos y hallar una diferencia mínima de los valores de niveles de potencia de recepción aceptable de los equipos de radio usados para tal fin.
\end{abstract}

Palabras clave: Cobertura, modelo de propagación, potencia, red malla, regresión.

\begin{abstract}
The used methodology is based on selecting and confines the workspace, determining the technical and regulatory criteria, the capacity of the equipments, recording of data, perform analytical processing, statistical analysis of the results and compare with indoor estimation models. The expected result is to obtain the coefficients of the mathematical model that approximates the power levels measured and calculated. The last step will be to confront the data with established models and find a minimum of the values of power levels of acceptable radio reception equipments used for this purpose.
\end{abstract}

Keywords: Coverage, mesh network, power, propagation model, regression.

\section{INTRODUCCIÓN}

Los bajos costos en la instalación y mantenimiento de redes implementadas como tipo malladas son una alternativa a las redes inalámbricas convencionales debido a que se mejoran los niveles de cobertura por potencia y se aumenta el número de clientes potenciales que pueden compartir los enlaces, sin embargo, los rendimientos de este tipo de redes dependen de las condiciones ambientales y la calidad de los protocolos de enrutamiento.
En el diseño y planificación de una red inalámbrica con línea o sin línea de vista, es importante verificar los elementos y factores de afectan los niveles de potencia de la señal tales como: la atenuación, la distorsión, el ruido, el multitrayecto, entre otros; debido a que existe una gran variabilidad de la señal por los efectos de los fenómenos físicos (la humedad, la presión, la altura y los cambios abruptos de temperatura) que dependen del ambiente y la región donde se desea 
implementar, también se debe considerar a interferencia debida a los elementos electrónicos externos que producen señales de radio frecuencia (RF) que afectan la frecuencia del espectro de trabajo seleccionado según (Rappaprt \& Sandhud, 1995).

Los efectos de varias fuentes de interferencia pueden ser estimados calculando la relación señal interferencia en el receptor de interés, considerando que existen fuentes cercanas a la frecuencia de portadora como es el caso del efecto de canal adyacente, causada por radiaciones electromagnéticas no deseadas haciendo que en el receptor disminuya la ganancia en potencia (Dobkin, 2002).

Una red multi saltos mallada consiste en enrutadores colocados en forma estacionaria estratégicamente para proporcionar una infraestructura distribuida a los clientes móviles, de acuerdo a (Osekowska, 2012), de manera que sea robusta, adaptable y flexible en el proceso de conectividad.

El objetivo del estudio es evaluar el rendimiento en la calidad de la potencia que está influenciada por muchos factores de propagación que afectan la señal, pero la eficacia de una red de este tipo se apoya en el protocolo de enrutamiento para redes malladas; protocolo híbrido de malla inalámbrica (HWMP); es un protocolo de enrutamiento de malla que combina la flexibilidad en la demanda de enrutamiento con topología proactiva árbol que permite una ruta óptima y eficiente; por medio de un conjunto de primitivas de protocolo, reglas de generación y procesamiento tomados del protocolo Distancia vectorial por demanda AD-hoc (AODV), dicho protocolo es adaptado para la capa 2 con el enrutamiento basado en la dirección y una métrica de coincidencia permitiendo mantener un enlace más estable (Batiste, 2011).

Lo anterior, se realiza con el fin de mejorar el nivel de cobertura de la red interna del Campus Universitario debido a que el aumento del tráfico de datos en horas picos hace que los enlaces se bloquean, existen áreas de trabajo en donde el nivel de potencia es muy deficiente generando retardos en la trasmisión de datos entre los equipos de la red, por tanto, se desea determinar las áreas en donde sea posible realizar una reubicación e instalación de estaciones base con el fin de aprovecharla funcionalidad y capacidad de enrutamiento del protocolo HWMP, para mejorar la cobertura y la calidad de la señal.

\section{MODELOS DE REFERENCIA DE PÉRDIDAS}

Las principales características de un entorno de propagación de RF en ambientes interiores con respecto de un entorno en exteriores, es el efecto multi trayecto que generalmente es más pronunciado en este último; según (Balanis, 2005), en un trayecto con línea de vista puede no existir, además, las características del entorno pueden cambiar drásticamente en tiempo o distancias muy cortas menores a los 100 metros.

Las paredes, puertas, muebles y las personas pueden causar la pérdida significativa de la señal. Las pérdidas de trayectoria cambian dramáticamente con el tiempo o la posición, debido a la cantidad de múltiples trayectos presentes, el movimiento de personas, equipos y/o puertas.

Cuando se considera un canal de propagación en interiores, es evidente que en muchos casos no hay una línea de visión directa entre el transmisor y el receptor. En tales casos, la propagación depende de: la reflexión, la difracción, la penetración de intensidad de potencia, la dispersión y la decoloración según planteamiento de (Cardama, 2004), mecanismos de propagación que degradan la señal. El retardo y la dispersión Doppler, suelen ser mucho menos significativos en un ambiente interior debido que las distancias son mucho más pequeñas y velocidades son más bajas en el transceptor portátil en comparación con ambientes en exteriores enunciado por (Seybold, 2005).

Con respecto a los modelos que se utilizan en este tipo de entornos, en general dominan los modelos sin línea de vista (NLOS), aunque no se deben descartar completamente los modelos con línea de vista (LOS). No obstante, aunque en general se basan todos ellos en medidas experimentales, existen algunos modelos teóricos que a pesar de su complejidad se deben considerar en estudios futuros, como es el caso de: El modelo de trazado de rayos y modelos con análisis de dominio temporal de diferencia finita, planteados por (Tuan, 2003), los cuales utilizan algoritmos matemáticos complejos para la síntesis de los resultados, pero que no son abordados en esta investigación.

La banda de frecuencias donde más se han realizado estudios para este tipo de modelos es la comprendida entre las frecuencias del orden de 1,8 $\mathrm{GHz}$ a $2,4 \mathrm{GHz}$, como es caso de los trabajos realizados con el modelo de COST-231 por medio de análisis simplificados como son: Modelos empíricos de banda estrecha: (Modelo de pendiente única, modelo de múltiples paredes y modelo de 
atenuación línea), Modelos empíricos de Banda ancha y Modelos deterministas (Modelo de lanzamiento de rayos, modelo de acercamiento de imagen). Para el caso de estudio se analizan los siguientes modelos:

\subsection{Modelo de pendiente única}

Es un modelo de referencia básica para este tipo de cálculos y sigue la expresión habitual de la propagación:

$$
\mathrm{L}_{(\mathrm{dB})}=\mathrm{L}_{0}-10 * \mathrm{n} * \log (\mathrm{d})
$$

Dónde Lo son las pérdidas de propagación a una distancia de referencia igual a 1 metro y $n$ es el índice de variación de la potencia con la distancia; parámetros, que dependen de la frecuencia y el entorno de propagación; con los valores calculados se minimiza el error cuadrático medio de los datos medidos experimentalmente. El valor de $d$ se expresada en metros.

\subsection{Modelo de múltiples paredes}

Es un modelo que precisa datos reales y exactos del entorno para deducir resultados fiables. A partir de ellos se obtienen datos que representan la realidad con bastante precisión. Es un modelo empírico que parte del modelo Motley del año 1990, planteado por (Sedin, 2004).

$$
\mathrm{L}_{(\mathrm{dB})}=\mathrm{Lo}+10 * \mathrm{n} * \log (\mathrm{d})+\sum_{\mathrm{i}=1}^{\mathrm{I}} \mathrm{K} * \mathrm{fi} * \mathrm{Lfi}+\sum_{\mathrm{j}=1}^{\mathrm{J}} \mathrm{K} * \mathrm{Wj} * \mathrm{LWj}
$$

Los parámetros representan:

\section{$d$ : Distancia en metros.}

Lo: Referencia de pérdidas a un metro de distancia que Motley indica de $37 \mathrm{~dB}$.

$n$ : Indicador de la variación de la potencia con la distancia (típico $\mathrm{n}=1$ y $L o=37 \mathrm{~dB}$, espacio libre).

$L$ fi: Factor de perdida por piso tipo $i$.

$L w j$ : Factor de perdida por pared tipo $j$.

$K$ fi: Número de pisos tipo $i$ atravesados.

$K$ wj: Numero de paredes tipo $j$ atravesadas.

$I$ : Número tipo piso, $J$ : Número tipo pared.

\subsection{Modelo Cost-231}

A través de estudios realizados por Hashemi (1993) se añaden modificaciones al modelo de Motley y como resultado de la combinación de otros modelos se propone un modelo semi - empírico por medio de una fórmula con resultados particulares alrededor de las medidas observadas por la atenuación de los suelos y su alinealidad.

$\mathrm{L}_{(\mathrm{dB})}=\mathrm{L}_{\mathrm{el}}+\mathrm{L}_{\mathrm{c}}+\sum_{\mathrm{j}=1}^{\mathrm{J}} \mathrm{Kwj}^{*} \mathrm{Lwj}^{*}\left\{\frac{(\mathrm{Kfi}+2)}{(\mathrm{Kfi}+1)}-b\right\} * \mathrm{Lfi}$

Donde Lel son las pérdidas en espacio libre, $L c$ es un factor constante y $\mathrm{b}$ es un factor empírico.

\subsection{Modelo UIT-R 8/1}

Tratando de simplificar el cálculo del modelo anterior sin perder su aumento de exactitud respecto del primero, se tiene que la expresión se puede compactar como:

$$
\mathrm{L}_{(\mathrm{dB})}=38 \mathrm{~dB}+30 * \log (\mathrm{d})_{(\mathrm{m})}+\mathrm{Lf}(\mathrm{p})
$$

Donde $\mathrm{L} f(p)$ es el factor de pérdidas de penetración en paredes y suelos, se representa como:

$\mathrm{Lf}(\mathrm{p})=15+4(\mathrm{p}+1)$

Donde $\mathrm{p}$ es el número de plantas entre transmisor y receptor.

\subsection{Método de long-distance}

Este modelo permite calcular las perdidas por trayectoria en ambientes interiores por la ley de la potencia.

$$
\mathrm{L}_{(\mathrm{dB})}=\operatorname{PL}(\mathrm{d} 0)+10 * \mathrm{n} * \log \left(\frac{\mathrm{d}}{\mathrm{d} 0}\right)+\mathrm{X} \sigma
$$

Para esta ecuación, $\mathrm{n}$ es el exponente de pérdidas que depende del tipo de ambiente y edificación y $X \sigma$ representa la variable aleatoria normal en $\mathrm{dB}$ debida a la desviación estándar.

\section{DISEÑO METODOLÓGICO EXPERIMENTAL}

\subsection{Análisis de los modelos de propagación en ambientes interiores}

Dentro del proceso de documentación se analizaron los diferentes tipos de modelos y métodos de propagación existentes para sistemas móviles que sirven como referencia para el tipo de frecuencia a trabajar, por tanto, se extrapolaron algunos valores y características del tipo de ambientes, condiciones físicas y geográficas del espacio de trabajo confinado.

Para el proceso de análisis y comparación se utilizan como referencia los siguientes modelos: El de pendiente única, de múltiples paredes, COST231, Modelo UIT-R 8/1 y el Modelo Log-Distance, debido a que son modelos empíricos que comparten condiciones particulares similares para este tipo de ambientes en interiores.

\subsection{Selección del área de trabajo.}

Consiste en la selección de un sector del campus universitario de la Universidad de Pamplona en Villa del Rosario Cúcuta N.S., el sistema de red es una configuración tipo mallada fija jerarquizada, 
toda la conectividad se realiza a través de una red LAN conmutada para realizar el control y la administración conjunta por medio de las direcciones MAC de cada uno de los equipos de radio, trabajando en la frecuencia de $2,4 \mathrm{Ghz}$ en el canal 11, con un trazado de tres rutas como aparece en la (Fig. 1), debido a que es un rango del espectro que más problemas presenta al momento de implementar una red inalámbrica se desea contrastar con los diferentes modelos existentes que han sido mencionados anteriormente e inferir sobre los posibles ajustes que se pueden realizar.

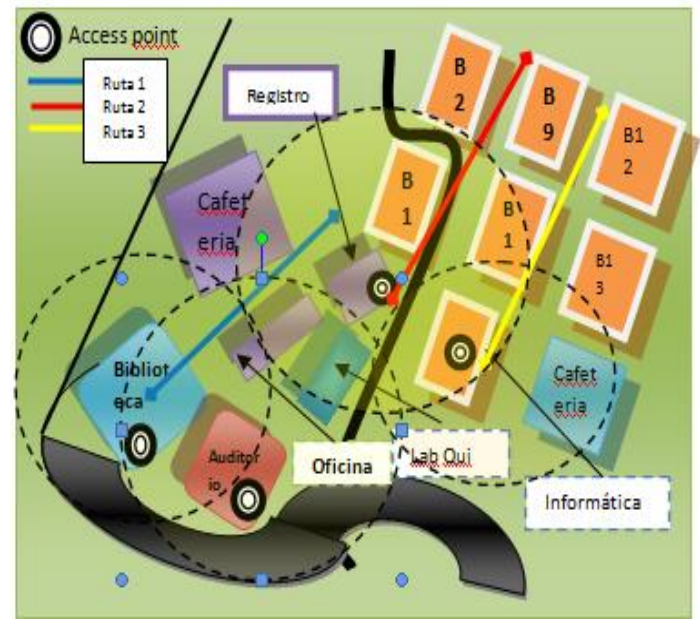

Fig. 1. Sector 1: área de trabajo.

La campaña de medidas se realiza teniendo en cuenta la proximidad a las fuentes de radiación y porque son las rutas que generan el mayor tráfico para realizar la toma de muestras de la intensidad de potencia que aporta cada equipo de radio hacia las estaciones móviles (EM) o clientes bajo condiciones de visibilidad mixta, (con línea de vista y sin línea de vista) debido a que el estudio de campo considera que las celdas comparten una gestión centralizada, por tanto, el análisis se realiza como una red mallada (red mesh), configurada como tipo Pico célula por la posición típica de las antenas de las estaciones base (EB) con cubrimiento de 100 metros de radio por celda en ambiente interior (montada por debajo del nivel máximo de los tejados), según la norma (UIT-R P.1411-7, 2013).

\subsection{Criterios técnicos y reglamentación}

Es importante considerar que en el ambiente en interiores para realizar las mediciones en presencia de condiciones abruptas por lo general, tienden a deteriorar el nivel de intensidad de señal esperado a medir, situación técnica que mejora haciendo una agrupación de mediciones con el propósito de obtener un promedio de potencia más confiable, para el estudio en particular se realizó la toma de 180 muestras durante 3 minutos de acuerdo a las especificaciones de la Recomendación UIT-R SM.378 que recomienda que: "salvo cuando existan limitaciones debidas al nivel de ruido del receptor, ruido atmosférico o interferencias externas, la precisión que cabe esperar en las mediciones de intensidad de campo es mejor que $\pm 2 \mathrm{~dB}$ para frecuencias inferiores a $30 \mathrm{MHz}$ y \pm 3 $\mathrm{dB}$ para frecuencias superiores a $30 \mathrm{MHz}$, de acuerdo a las disposiciones del (Manual de Comprobación, 2011).

Los equipos controlados por microprocesador pueden conmutar frecuencias y antenas, si es preciso, descargar la energía residual de la banda de paso del receptor, efectuar mediciones sobre una nueva frecuencia, incluyendo la aplicación de factores de corrección, y registrar todos los resultados en forma digital en cuestión de décimas de milisegundos. Los resultados deben registrarse en la memoria de un computador debido a la gran cantidad de datos obtenidos en un tiempo muy corto".

También se debe considerar que "La posible precisión en condiciones normales con instalaciones portátiles o móviles hace que la precisión lograda no satisface en todos los casos lo dispuesto en la recomendación UIT-R 378, pero, aun así, la capacidad de recepción omnidireccional" hace de la antena de $\lambda / 4$ un instrumento útil para realizar mediciones de campo, para el caso en estudio y bajo los estándares de la norma (UIT-R P.1406, 2012).

Para medir la distribución en el tiempo de la intensidad de campo pueden realizarse mediciones de corta duración y de larga duración con un sistema instalado permanentemente, por ejemplo, en una estación fija o situada en un contenedor. Las mediciones pueden ser continuas o bien repetirse a intervalos regulares, permitiendo la observación de varias frecuencias. Los tiempos de medición muy largos, exigen realizar una verificación frecuente de la calibración. También es posible realizar mediciones a largo plazo utilizando equipos de medición manuales junto con registradores de datos.

En la comprobación técnica de la ocupación del canal en el espectro medido se mantienen los criterios de la recomendación (UIT-R SM.1392, 2012), para el caso de los requisitos esenciales para los países en desarrollo, la cual establece que la recolección de datos de ocupación del espectro, 
incluidos el nivel de la señal de canales individuales, para la asignación de frecuencias, es fundamental realizar mediciones permanentes. Estas mediciones se realizan normalmente mediante un método automatizado.

Para el caso de estudio con fines prácticos el análisis de la cobertura de la comprobación técnica se logra de forma flexible por medio de: Sistemas de comprobación técnica económicos para bandas/señales especiales (por ejemplo, sistemas de comprobación técnica para la banda (Industrial, científica y médica, ISM) en la frecuencia de 2,4 $\mathrm{GHz}$, con técnicas móviles y portátiles como lo cita la norma (UIT-R SM. 2039, 2013).

\subsection{Características técnicas de los equipos}

Los equipos de radio utilizados emulan una tecnología mallada predecible que es de tipo fija (normalmente las redes malladas son dinámicas) debido a que soporta hasta 4 enlaces inalámbricos ascendentes (Wireless uplink) desde un equipo maestro habilitado para controlar los radios clientes o hijos por medio de conexiones cableadas a una red de área local (LAN).

El software de administración permite que los radios estén ubicados estratégicamente sobre rangos de distancias de cobertura aproximadas de hasta 120 metros para configurar celdas que están interconectadas a nivel de capa 2 del modelo TCP/IP dentro del mismo segmento de red en un mismo canal, la aplicación realiza la detección por control de acceso al medio (MAC) para identificar cuando un usuario móvil cambia entre los diferentes puntos de acceso.

El balanceo de carga, la distribución de potencia y el acceso de los usuarios se realiza de forma que cada cliente este enlazado a cualquiera de los cuatro puntos de acceso para mantener un nivel de señal de potencia adecuado. La estación base es un radio Unifi Access Point Outdoor (UAP outdoor) de (Ubiquiti Networks, 2015), con dos antenas omnidireccionales cada una de $6 \mathrm{dBi}$, configurados con potencia máxima de $27 \mathrm{dBm}$, sensibilidad -96 $\mathrm{dBm}$, ancho banda canal 20/40Mhz, un sistema mimo 2x2 los radios están ubicados a 2,50 metros de alturas en los muros, la estación móvil tiene una ganancia de $2 \mathrm{dBm}$.

\subsection{Realizar medidas del nivel de potencia}

El recorrido de las distancias entre el transmisor y el receptor se hace cada 5 metros (40 longitudes de onda de la frecuencia de trabajo) haciendo el recorrido, para cada una de las rutas hasta lograr un nivel de potencia óptimo aplicando las recomendaciones de la UIT. Se encienden los radios por 15 minutos antes de empezar a realizar las mediciones para evitar error de ruido térmico o electrónico al iniciar la toma de los datos. Se realizan los recorridos correspondientes tomando muestras cada 180 segundos durante 2 secciones en días diferentes, de acuerdo a (Herrera y Pérez, 2008), pero manteniendo un horario y unas condiciones climáticas relativamente iguales.

\subsection{Registro de datos}

La captura y medición de los niveles de potencia recibidos en el radio se guardan en un equipo de cómputo para realizar el análisis estadístico para la frecuencia de trabajo de $2,4 \mathrm{GHz}$.

El proceso de depuración de los datos consiste en filtrar la información redundante, es decir, solo se selecciona el nombre del radio que emite el nivel de potencia en $\mathrm{dBm}$ en el sector donde radia para luego obtener las gráficas que relacionan potencia con respecto a distancia. El procedimiento consiste en: Captura mediante un software libre InSSDIer el nivel de potencia que se guarda en tiempo real en un archivo tipo log, después se importa dicho archivo a una hoja electrónica y se filtran los datos de interés, como son: La dirección MAC, nombre del radio, nivel de potencia, fecha y hora, después se importan los datos filtrados al software estadístico, para realizar el análisis correspondiente, obtener las funciones y datos estadísticos descriptivos; en este caso se trabajó con la media, como medida de tendencia central y los estadístico de dispersión: la desviación típica y la varianza, por medio de los cuales se logra un nivel de confianza de las mediciones como se detallan más adelante en las tablas.

\subsection{Procedimiento analítico}

Generar una ecuación con los datos obtenidos de los niveles de potencia medidos con la ayuda del software estadístico SPSS16 de la empresa (IBM , 2015), aplicando el método de regresión no lineal se obtiene una función estimada, como se observa en el siguiente ejemplo (Fig. 2). 


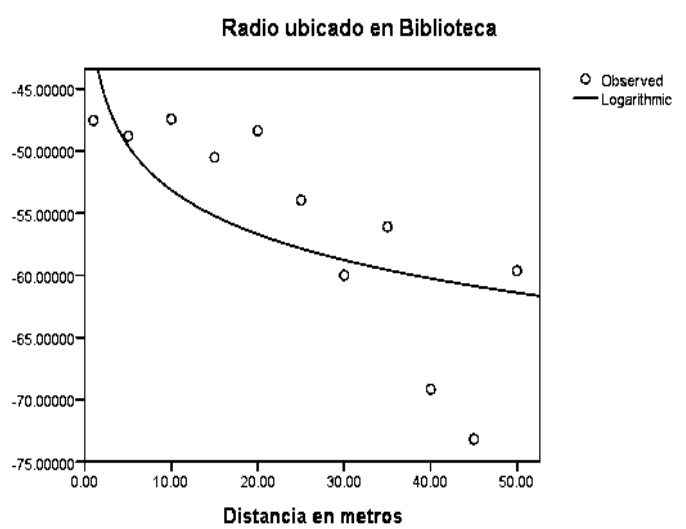

Fig. 2: Curva del modelo de regresión no lineal. Diseño con software SPSS ® Versión 16

Simultáneamente se obtienen los valores estadísticos como se muestra en la tabla 1, para obtener la expresión analítica.

Tabla 1: Coeficientes resultado del análisis de regresión. Diseño con software SPSS ${ }^{\circledR}$ Versión 16

\begin{tabular}{|c|c|c|c|c|c|}
\hline & \multicolumn{2}{|c|}{$\begin{array}{l}\text { Unstandardized } \\
\text { Coefficients }\end{array}$} & \multirow{2}{*}{$\begin{array}{l}\text { Standar } \\
\text { dized } \\
\text { Coeffici } \\
\text { ents } \\
\text { Beta }\end{array}$} & \multirow[t]{2}{*}{$t$} & \multirow[t]{2}{*}{ Sig. } \\
\hline \multirow{3}{*}{$\begin{array}{l}\ln \\
(\text { Distanci }=\mathrm{d} \\
(\text { Consta })=\mathrm{c}\end{array}$} & B & $\begin{array}{l}\text { Std. } \\
\text { Error }\end{array}$ & & & \\
\hline & -5.134 & 1.862 & -.677 & -2.757 & .022 \\
\hline & $\begin{array}{l}- \\
41.322\end{array}$ & 5.676 & & -7.280 & .000 \\
\hline
\end{tabular}

Un ejemplo del resultado del proceso de regresión no lineal, es la ecuación que se deduce con los coeficientes de la tabla 1 :

$$
\mathrm{y}_{(\mathrm{dBm})}=-41,322-5,134 * \ln (\mathrm{d})
$$

La expresión de la ecuación 7, muestra que el comportamiento de este tipo de ambientes es de tipo logaritmo como producto de que la potencia de recepción varía con el logaritmo de la distancia a medida que se realizan las medidas en cada punto, los datos después son probados y comparados con los modelos de propagación existentes para ambientes interiores. Es de resaltar que los métodos de estimación en radioenlaces fijo, han partido siempre de las pérdidas de propagación en espacio libre.

\section{RESULTADOS}

Los modelos de propagación buscan predecir el nivel de intensidad de señal en forma estocástica de acuerdo al área de cobertura estimada, a primera vista podría parecer que la predicción en ambientes interiores puede resultar sencilla pero no es así, por el efecto que producen muchos factores físicos y tipos de construcción, por pequeños que parezcan.

Los resultados de las mediciones obtenidas se tabularon en una hoja electrónica para obtener los promedios de las mediciones junto con los datos de la estadística descriptiva como se observa en la tabla 2 (a), (b) y (c), para cada uno de los tres radios de acuerdo a su ubicación. La información se obtuvo de la agrupación de las mediciones de la potencia promedio medida cada 5 metros (en diferentes horas y rutas), con la toma de 180 muestras en cada punto durante 3 minutos, los datos se filtran, debido a que la herramienta toma muestras de potencia de otros radios cercanos por al canal del espectro medido.

\section{Tabla 2(a): Promedio potencias obtenidas en el} radio Biblioteca

\begin{tabular}{l|cccc}
\hline $\begin{array}{l}\text { Ubicación } \\
(\text { metros })\end{array}$ & $\begin{array}{l}\text { Biblioteca } \\
(\mathrm{dBm})\end{array}$ & $\begin{array}{l}\text { Sala } \\
\text { Informática } \\
(\mathrm{dBm})\end{array}$ & $\begin{array}{l}\text { Auditorio } \\
(\mathrm{dBm})\end{array}$ & $\begin{array}{l}\text { Registro } \\
(\mathrm{dBm})\end{array}$ \\
\hline 1 & $-47,55$ & $-69,53$ & $-54,04$ & $-67,47$ \\
5 & $-48,79$ & $-68,55$ & $-60,92$ & $-75,70$ \\
10 & $-47,43$ & $-86,19$ & $-60,01$ & $-83,94$ \\
15 & $-50,51$ & $-67,57$ & $-55,62$ & $-67,75$ \\
20 & $-48,37$ & $-83,49$ & $-71,91$ & $-73,17$ \\
25 & $-53,96$ & $-64,10$ & $-59,82$ & $-63,41$ \\
30 & $-60,01$ & $-71,83$ & $-78,27$ & $-70,11$ \\
35 & $-56,08$ & $-62,32$ & $-63,14$ & $-59,53$ \\
40 & $-69,18$ & $-72,32$ & $-82,51$ & $-57,41$ \\
45 & $-73,19$ & $-78,35$ & $-74,64$ & $-65,60$ \\
50 & $-59,65$ & $-59,27$ & $-74,64$ & $-60,21$ \\
\hline
\end{tabular}

En esta tabla 2 (a) se observan los aportes de la potencia de los radios: Sala informática, auditorio y registro dentro la misma celda formada por el radio ubicado en la biblioteca, que contribuyen a mejorar el nivel de potencia.

En este caso de tabla 2 (b) se muestran las contribuciones la potencia de los radios: Biblioteca, Sala informática y auditorio con contribuciones de niveles de potencia al radio ubicado en registro.

Tabla 2(b): Promedio potencias obtenidas en el radio Registro

\begin{tabular}{l|lrrr}
\hline $\begin{array}{l}\text { Ubicac } \\
\text { ión } \\
\text { (metros) }\end{array}$ & $\begin{array}{l}\text { Bibliote } \\
\text { ca } \\
(d B m)\end{array}$ & $\begin{array}{l}\text { Sala } \\
\text { Informáti } \\
\text { ca } \\
(d B m)\end{array}$ & $\begin{array}{l}\text { Auditor } \\
\text { io } \\
(d B m)\end{array}$ & $\begin{array}{l}\text { Regist } \\
\text { ro } \\
(d B m)\end{array}$ \\
\hline 1 & $-58,37$ & $-58,41$ & $-58,37$ & $-58,37$ \\
5 & $-86,37$ & $-66,33$ & $-63,25$ & $-57,60$ \\
10 & $-69,17$ & $-48,07$ & $-65,69$ & $-58,22$ \\
\hline
\end{tabular}


Revista Colombiana de Tecnologías de Avanzada

\begin{tabular}{l|llll}
\hline 15 & $-69,17$ & $-46,63$ & $-65,69$ & $-58,22$ \\
20 & $-86,60$ & $-74,79$ & $-83,96$ & $-78,10$ \\
25 & $-68,57$ & $-59,25$ & $-64,95$ & $-63,67$ \\
30 & $-70,89$ & $-61,53$ & $-69,94$ & $-69,81$ \\
35 & $-77,50$ & $-82,27$ & $-85,86$ & $-85,78$ \\
40 & $-68,44$ & $-67,81$ & $-87,61$ & $-67,64$ \\
45 & $-81,65$ & $-84,25$ & $-88,20$ & $-89,92$ \\
\hline
\end{tabular}

En la tabla 2 (c) se detallan los aportes de la potencia de los radios: Biblioteca, auditorio $\mathrm{y}$ registro dentro de la celda. Después los datos de las tres tablas referidas anteriormente son importados al programa SPSS Versión 16 para realizar el análisis estadístico de los cuales se extraen los valores estadísticos más significativos en las tablas 3 (a), (b) y (c) de forma independiente.

Tabla 2(c):: Promedio potencias obtenidas en el radio Sala de informática

\begin{tabular}{l|lcll}
\hline $\begin{array}{l}\text { Ubicaci } \\
\text { ón } \\
\text { (metros) }\end{array}$ & $\begin{array}{l}\text { Bibliote } \\
\text { ca } \\
(d B m)\end{array}$ & $\begin{array}{l}\text { Sala } \\
\text { Informáti } \\
\text { ca } \\
(d B m)\end{array}$ & $\begin{array}{l}\text { Auditor } \\
\text { io } \\
(d B m)\end{array}$ & $\begin{array}{l}\text { Regist } \\
\text { ro } \\
(d B m)\end{array}$ \\
\hline 1 & $-69,18$ & $-48,83$ & $-66,81$ & $-59,93$ \\
5 & $-77,66$ & $-59,71$ & $-72,99$ & $-70,87$ \\
10 & $-72,80$ & $-58,68$ & $-70,02$ & $-70,68$ \\
15 & $-75,23$ & $-57,45$ & $-62,72$ & $-65,02$ \\
20 & $-75,48$ & $-61,97$ & $-73,00$ & $-71,49$ \\
25 & $-91,00$ & $-71,62$ & $-82,15$ & $-82,01$ \\
30 & $-82,30$ & $-80,66$ & $-82,17$ & $-84,49$ \\
35 & $-54,46$ & $-61,75$ & $-62,80$ & $-67,52$ \\
40 & $-75,69$ & $-75,63$ & $-84,74$ & $-89,56$ \\
\hline
\end{tabular}

En las medidas estadísticas de la radio estación base Biblioteca se aprecia que el nivel de desviación estándar de la potencia de los radios cercanos, oscila entre 7,842 $\mathrm{dBm}$ y $9,788 \mathrm{dBm}$ en la tabla 3 (a).

De forma similar para la radio estación base Registro el valor del nivel de desviación estándar de la potencia de los radios cercanos, oscila entre el rango de $9,124 \mathrm{dBm}$ a $13,733 \mathrm{dBm}$ en la tabla 3 (b).

Tabla 3 (a): Descriptive Statistics Biblioteca. Diseño con software SPSS $®$ Versión 16

\begin{tabular}{l|cccccc}
\hline & St & & Statisti & Std. & Std & Var \\
& $a t i$ & & $c$ & Error &. & ian \\
& sti & & & & De & $c e$ \\
& $c$ & & & & via & \\
& & & & & & tio \\
& & & & & $n$ & \\
\hline Bibliote & 11 & $-6.147 \mathrm{E} 2$ & -5.5884 & 2.676 & 8.8 & 78.7 \\
ca & & & & & 76 & \\
Auditori & 11 & $-7.353 \mathrm{E} 2$ & -6.6866 & 2.951 & 9.7 & 95.8 \\
\hline
\end{tabular}

\begin{tabular}{l|rrrrrr}
\hline o & & & & & 88 & \\
Sala & 11 & $-7.835 \mathrm{E} 2$ & -7.1228 & 2.563 & 8.5 & 72.3 \\
Informá & & & & & 03 & \\
tica & & & & & & \\
Registro & \multirow{2}{*}{11} & $-7.443 \mathrm{E} 2$ & -6.7664 & 2.364 & 7.8 & 61.5 \\
& & & & & 42 & \\
& & & & & & \\
\hline
\end{tabular}

Tabla 3(b): Descriptive Statistics Registro. Diseño con software SPSS ® Versión 16

\begin{tabular}{|c|c|c|c|c|c|c|}
\hline & $\begin{array}{c}\text { Sta } \\
\text { tis }\end{array}$ & Statistic & $\begin{array}{l}\text { Statist } \\
i c\end{array}$ & $\begin{array}{c}S t d \\
\dot{E} \\
\text { Err } \\
\text { or }\end{array}$ & $\begin{array}{c}\text { Std. } \\
\text { Devi } \\
\text { ation }\end{array}$ & $\begin{array}{c}\text { Varian } \\
\text { ce }\end{array}$ \\
\hline $\begin{array}{l}\text { Bibliote } \\
\mathrm{ca}\end{array}$ & 11 & -820.34 & -74.57 & $\begin{array}{r}2.7 \\
51\end{array}$ & 9.124 & 83.2 \\
\hline $\begin{array}{l}\text { SalaInf } \\
\text { orma }\end{array}$ & 11 & -735.23 & -66.83 & $\begin{array}{r}4.1 \\
40\end{array}$ & $\begin{array}{r}13.73 \\
3\end{array}$ & 188.6 \\
\hline $\begin{array}{l}\text { Auditor } \\
\text { io }\end{array}$ & 11 & -822.31 & $\begin{array}{r}- \\
74.755 \\
2\end{array}$ & $\begin{array}{r}3.6 \\
12\end{array}$ & $\begin{array}{r}11.98 \\
1\end{array}$ & 143.5 \\
\hline $\begin{array}{l}\text { Registr } \\
\mathrm{o}\end{array}$ & 11 & -776.08 & $\begin{array}{r}- \\
70.552 \\
5\end{array}$ & $\begin{array}{r}3.8 \\
98\end{array}$ & $\begin{array}{r}12.92 \\
9\end{array}$ & 167.1 \\
\hline
\end{tabular}

Finalmente en la tabla 3 (c), en la estación base Sala informática se detalla que el nivel de desviación de la potencia de los radios cercanos oscila entre $8,385 \mathrm{dBm}$ a 9,999 $\mathrm{dBm}$ como limites contributivos de forma que algebraicamente se puede incrementar o disminuir el valor real de la potencia recibida.

El aplicativo SPSS arroja los parámetros con los cuales se obtienen las ecuaciones no lineales más significativas para los tres radios analizados como se muestran en las figuras 3 (a), (b) y (c) de acuerdo a los niveles de potencias recibidos en las tres rutas trazadas que hacen parte de la celda de acuerdo a (Campos, 2004).

Tabla3 (c): Descriptive Statistics Sala informática. Diseño con software SPSS $®$ Versión 16

\begin{tabular}{l|ccrrrr}
\hline & $S$ & Sum & Sta & Std. & Std. & Vari \\
& $t$ & Statistic & tist & Erro & Devi & ance \\
& $a$ & & ics & $r$ & ation & \\
& $t$ & & & & & \\
& $i$ & & & & & \\
\hline Bibliot & 9 & $-6.737 \mathrm{E} 2$ & - & 3.28 & 9.85 & 97.1 \\
eca & & & 7.4 & 59 & 7 & \\
SalaInf & 9 & $-5.762 \mathrm{E} 2$ & - & 3.33 & 9.99 & 99.9 \\
orm & & & 6.4 & 31 & 9 & \\
Auditor & 9 & $-6.573 \mathrm{E} 2$ & - & 2.79 & 8.38 & 70.3 \\
io & & & 7.3 & 52 & 5 & \\
& & & 0 & & & \\
Registr & 9 & $-6.615 \mathrm{E} 2$ & - & 3.25 & 9.75 & 95.2 \\
o & & & 7.3 & 24 & 7 & \\
& & & 5 & & & \\
\hline
\end{tabular}


Al realizar las inferencias sobre un modelo logarítmico se deben incluir las variables y parámetros que afectan el comportamiento de la representación analítica en el proceso de la ecuación que va a representar en forma sintética los resultados obtenidos en campo, (Fig. 3.a) se observa que el coeficiente de determinación $\mathrm{R}^{2}$ es sencillamente el cuadrado del coeficiente de correlación, que expresado en porcentaje permite comparar que tan asociada esta la variable dependiente de la independiente.

En el caso del aporte significativo de potencia en $\mathrm{dBm}$ a los clientes que hacen parte de la red, se destaca que el radio ubicado en sala de informática aporta un $62,15 \%$ de señal mejor que su asociado ubicado en la Registro $(49,42 \%)$ como el más próximo y que podría haber contribuido con mayor nivel de potencia para el área de influencia durante todo el recorrido.

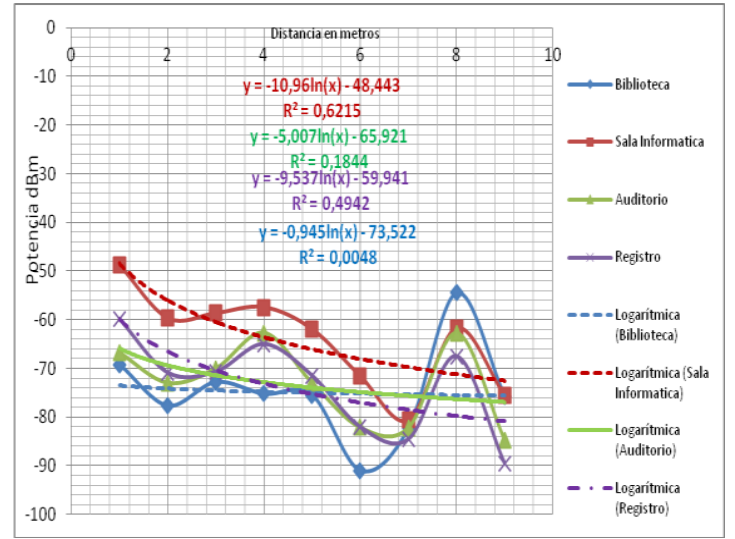

Fig. 3 (a): Curvas del modelo de regresión no lineal en sala de informática.

En el caso del Radio ubicado en Registro se presenta un aporte significativo de potencia del radio ubicado en el Auditorio con 67,46\%, (Fig. 3.b) en esta ruta se presentan más variaciones debido a las estructuras de cemento y los obstáculos en las aéreas de los pasillos, el radio de Registro propiamente radiando a su máxima potencia no garantiza un buen nivel de potencia superior al 56,22\%, lo cual hace que sea ineficiente.

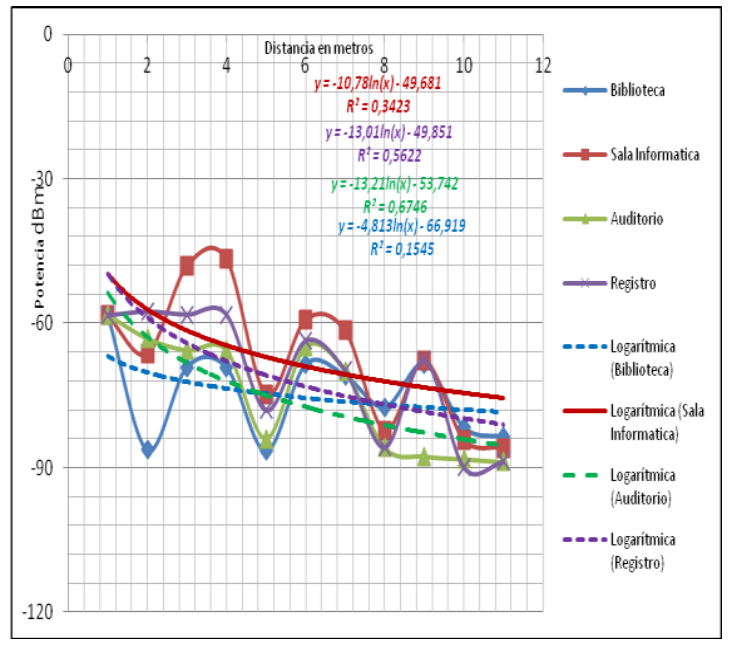

Fig. 3 (b): Curvas del modelo de regresión no lineal en Registro.

En la (Fig. 3.c), se aprecia que los niveles de potencia recibidos no sobrepasan el $60 \%$ haciendo que la medida de la bondad de ajuste del modelo de regresión no sea tan optimo como se esperaba, esto debido al efecto de las estructuras metálicas de la biblioteca, la densidad de árboles y los muros causan fenómenos de dispersión, refracción y difracción que afectan la intensidad de la señal.

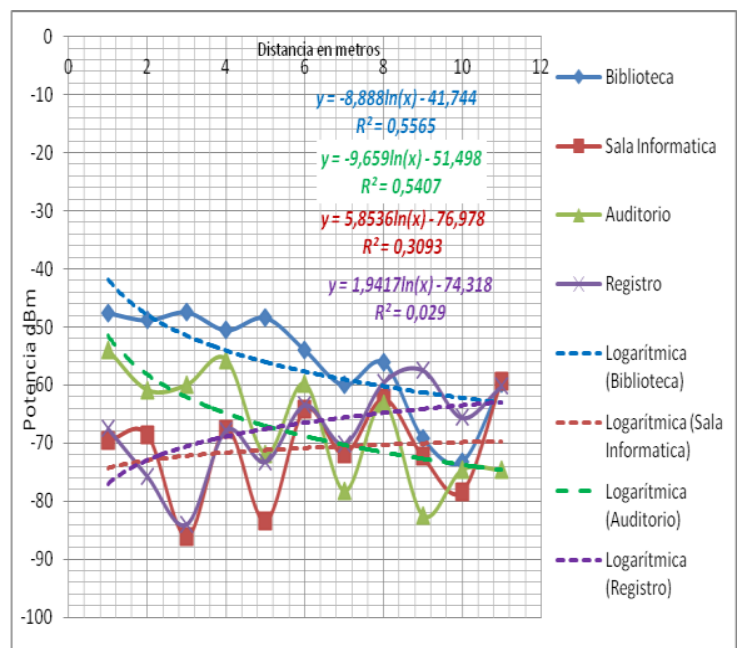

Fig.3(c ): Curvas del modelo de regresión no lineal en Biblioteca.

También se resalta que los cambios bruscos de señal con picos de potencia significativos hace que la curva de tendencia tenga una variación muy dispersa de la señal y el nivel de confiabilidad no sea muy eficiente, como el modelo de red implementado es de tipo malla se debe realizar una reconfiguración de la red basado en un modelo más robusto semejante a cómo trabaja un sistema móvil celular, de manera que se configure una pico celda 
que garantice un nivel de potencia más aceptable, inicialmente colocando antenas sectoriales y reubicando los radios para concentrar la mayor directivita y ganancia, sin necesidad de aumentar la potencia de los equipos transmisores.

Aunque, la calidad del modelo para predecir los resultados esperados o futuros se basan en el coeficiente de determinación, en general; la calidad de la ecuación que modela los sistemas en interiores sin línea de vista están por encima del $60 \%$, lo cual, permite que los enlaces de comunicación entre las estaciones bases y los móviles sean estables, pues, el nivel de sensibilidad máximo ofrecido por el fabricante de los equipos alcanzan los $-96 \mathrm{dBm}$, valor que en ningún momento fue superado en las mediciones.

Por otra parte, los enlaces se mantienen operativos porque la red mallada es predecible y el control centralizado hace que no se presente el fenómeno de traspaso de celda de forma permanente, pero la distancia de cobertura queda limitada a máximo 50 metros, es decir, el área de cobertura de la celda se reduce en un $50 \%$ de la distancia sugerida por el fabricante. En la confrontación con los modelos estadístico y semi empíricos y tomando como ejemplo, la ecuación del modelo obtenido para Sala de informática (Fig. 4), sé que tiene un comportamiento similar al modelo de pendiente y los niveles de pérdidas de potencias están dentro del umbral de sensibilidad permitiendo un comportamiento aceptable.

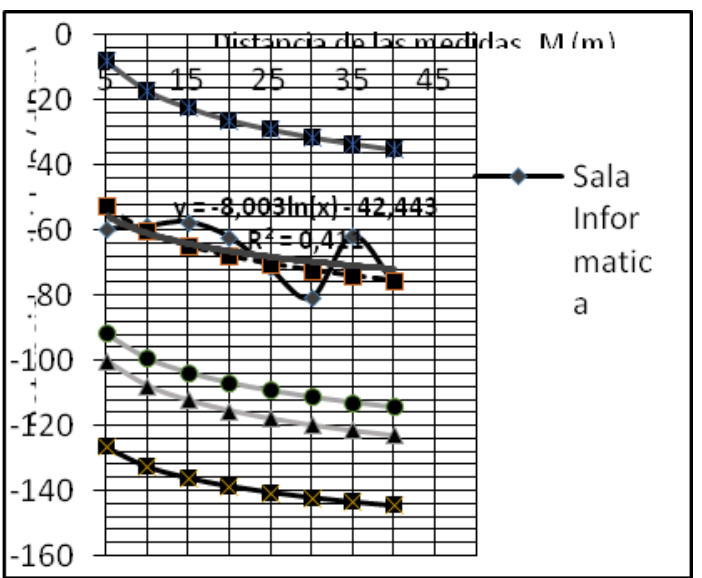

Fig. 4: Comparación modelos en ambientes interiores Radio informática.

\section{CONCLUSIONES}

En el proceso de mediciones se consideraron las tomas de las muestras en jornadas de más de una hora con el propósito de obtener valores de intensidad de potencia que no estén sesgados de la realidad, teniendo en cuenta el desvanecimiento de pequeña escala a nivel de pico celdas en donde se presentan desvanecimientos rápidos debidos a cambios de intensidad de la señal en pequeñas distancias y los efectos de dispersión debidos a la multi-trayectoria.

El modelado de propagación en interiores se complica por la gran variabilidad en: la construcción del diseño, materiales de construcción, el movimiento de personal, la densidad de árboles, las estructuras arquitectónicas y demás. Con una estadística descriptiva se realizan los ajustes a los datos empíricos para proporcionar un modelo que puede proporcionar una representación razonable, siempre y cuando se consideren aspectos más hostiles por el tipo de ambiente.

En la confrontación de los modelos en interiores de acuerdo a la figura 6 , el modelo más aproximado para los valores obtenidos por mediciones de campo para la frecuencia de $2,4 \mathrm{Ghz}$, es el modelo de pendiente única, aunque éste, no considera los factores y elementos que afectan la señal, como si se observa en el modelo Log-Distance y modelo de múltiples paredes; los modelos COST-231 y UIT-R $8 / 1$, están muy dispersos de valores reales y no se ajustan de manera eficiente a datos prácticos.

Para los demás radios el comportamiento es similar cuando se realizaron las mismas comparaciones.

Al momento de realizar una implementación de este tipo de red en ambientes interiores es clave destacar el valor de la desviación estándar de las mediciones porque aportan de manera sustancial al nivel de potencia que se desea: predecir, medir y calcular, de forma que se obtenga una diferencia mínima de los valores de sensibilidad que tiene establecido el hardware del equipo de radio.

En el proceso de las jornadas de medición las normas de la ITU recomiendan que se deban considerar las variaciones causadas por la absorción, dispersión y multi trayectoria causantes de las pérdidas en potencia para realizar los ajustes de los parámetros en un ambiente interior.

El protocolo de la red HWMP permite la combinación de métricas de ruteo y técnicas de ruteo basadas en árbol en estado proactivo, es decir, que conoce a los router vecinos agilizando el proceso de transmisión de las tramas a nivel de 
capa de enlace de datos con el manejo dinámico de las direcciones MAC de los equipos que están dentro de la red, pero, se presenta una disminución del rendimiento debido a que se sacrifica la distancia por los factores externos y la no disponibilidad de línea de vista.

\section{REFERENCIAS}

Campos Velázquez D, (2014), Cobertura dentro de construcciones para comunicaciones personal móviles vía satélite, Universidad de Puebla, Consultada:

http://catarina.udlap.mx/u_dl_a/tales/document os/lem/campos_v_da/capitulo_4.htm1.

Dobkin D., Indoor propagation issues for wireless LANs, RF Design Magazine, September 2002, pp. $40-46$.

Hashemi H., (2010). The Indoor Radio Propagation Channel, Proceedings of the IEEE. VOL 81, NO. 7, pp.943-968.

Herrera R., Jorge \& Pérez N., (2008), Modelo de propagación en ambientes exteriores para sistemas inalámbricos operando en la banda de frecuencias de $2.4 \mathrm{GHZ}$, Revista Colombiana de Tecnologías de Avanzada, Vol.2, N 12, pp. 114-124.

IBM SPSS Regression 20, Copyright IBM Corporation,(2011), Consultado: agosto 20 de 2015.

http://www.csun.edu/sites/default/files/regressi on20-32bit.pdf.

Manual de comprobación técnica del espectro, oficina de radiocomunicaciones, UIT Edición, (2011). Pp.103-207. Osekowska E.et al., (2012). Impact of Propagation Factors on Routing Efficiency in Wireless Mesh Networks: A Simulation-based Study,: The Eleventh International Conference on Networks, Copyright (c) IARIA,. ISBN: 978-161208-183-0, pp. 28-33.
Rappaport Theodore S. (2002) Wireless Communications Principles and practice, Segunda edición, Prentice Hall, United State of America, pp.55-89.

Rappapon Theodore S., Sandhu S., (1995). Radio wavepropagation for emerging wireless personal communication systems, Wireless Personal Communications, Springer Science+Business Media. ISBN 978-1-47572368-7, pp.1-29.

Recomendación UIT-R SM.1392, (2012). Requisitos esenciales para una estación de comprobación técnica del espectro para países en desarrollo, pp.1-7.

Recomendación UIT-R P.1406, (2013). Aspectos de la propagación relativos al servicio móvil terrestre terrenal en las bandas de ondas métricas y decimétricas. Pp.12-38.

Recomendación UIT-R P.1411-7, (2013). Propagation data and prediction methods for the planning of short-range outdoor radio communication systems and radio local area networks in the frequency range $300 \mathrm{MHz}$ to $100 \mathrm{GHz}, \mathrm{P}$ Series Radiowave propagation, pp.4-32.

Recomendación UIT-R SM.2039, (2013). Evolución de la comprobacióntécnica del espectro, Serie SM, Gestión del espectro.pp.16.

Sendín Escalona A., (2004). Fundamentos de los sistemas de comunicaciones móviles: Evolución y tecnologías,España: McGraw Hill, ISBN 84-481-4027-3,pp.62-98.

Seybold J., (2005). Introduction to RF propagation, editorial John Wiley, pp.209-215.

Ubiquiti Networks, User guide, Amp Outdoor,(2015), Consultada: http://dl.ubnt.com/guides/UniFi/UniFi_AP_Out door_UG.pdf. 